\title{
Detección del hongo defoliador Phaeocryptopus gaeumannii en plantaciones de Pseudotsuga menziesii de Valdivia, Chile
}

\author{
Detection of the needle cast fungus Phaeocryptopus gaeumannii in \\ plantations of Pseudotsuga menziesii of Valdivia, Chile
}

\begin{abstract}
Moisés Osorio O.
Universidad Austral de Chile, Facultad de Ciencias Forestales, Instituto de Silvicultura, casilla 567, Valdivia, Chile, Tel.: 56-63-221741, fonofax: 56-63-221228, mosorio@uach.cl
\end{abstract}

\begin{abstract}
SUMMARY
A situation of abnormality in plantations of Douglas-fir (Pseudotsuga menziesii) in the environs of Valdivia, Chile, was reported, which motivated a sanitary prospection. Stands of Douglas-fir of 2 to 34 years old and seedlings of a nursery were analyzed. It was stated that in trees of all these ages the foliage showed variable symptoms, from the normal green colour, until the brown yellow. By means of a magnifying glass, numerous very small spherical structures of black colour on the underside of infected needles were observed, aligned throughout the stomatics bands, which altogether gave a blackish aspect or of soot. These structures were in needles of trees of all the prospected ages and all showed symptomatological range in them. In the most extreme cases, the crown of the trees presented defoliations, and only needles of the last vegetative period were present. The microscopic analyses allowed to determine that the structures corresponded to ascocarps (pseudothecia) of the Phaeocryptopus gaeumannii fungus, recognized as pathogenic foliar of the Douglas-fir. These meaning of the presence of this agent in the other regions of the world is reviewed and beginning of mutidisciplinary studies set out for Chile to approach the problem is proposed.
\end{abstract}

Key words: swiss needle cast, Phaeocryptopus gaeumannii, Pseudotsuga menziesii.

\section{RESUMEN}

Se reportó una situación de anormalidad en plantaciones de pino oregón (Pseudotsuga menziesii) en los alrededores de Valdivia, Chile, lo que motivó la realización de una prospección sanitaria. Se analizaron rodales de pino oregón de 2 a 34 años de edad y también plantas de vivero. Se constató que en árboles de todas estas edades el follaje mostraba síntomas variables que iban desde el color verde normal, hasta el amarillo-pardo. Mediante una lupa se observaron en el envés de las acículas numerosas estructuras globosas, muy pequeñas, de color negro, alineadas a lo largo de las bandas estomáticas, las que en conjunto daban un aspecto negruzco o de hollín. Estas estructuras se encontraban en acículas de árboles de todas las edades prospectadas y de toda la gama sintomatológica manifestada en ellos. En los casos más extremos, la copa de los árboles presentaba defoliación, permaneciendo sólo las acículas del último período vegetativo. Los análisis microscópicos permitieron determinar que tales estructuras correspondían a ascocarpos (pseudotecios) del hongo Phaeocryptopus gaeumannii, reconocido como patógeno foliar del pino oregón. Se reseña el significado de la presencia de este agente en otras regiones del mundo y se propone para Chile el inicio de estudios multidisciplinarios para abordar el problema.

Palabras clave: defoliación, Pseudotsuga menziesii, Phaeocryptopus gaeumannii.

\section{INTRODUCCIÓN}

El pino oregón (Pseudotsuga menziesii (Mirb.) Franco) es una especie altamente apreciada tanto nacional como internacionalmente, debido a las características tecnológicas y belleza de su madera, a lo estético de su arquitectura y a su rápido crecimiento. Como especie exótica ha sido plantada en varios países de Europa Occidental, como también en Japón, Nueva Zelanda, Australia, Argentina, Turquía, e incluso en otros estados del propio Estados Unidos (Capretti et al. 1984, Davel 1998, INFOR-CONAF 1998, Quiroz y Rojas 2003, Hood 2004, Schütt et al. 2004). Ingentes esfuerzos privados y públicos se han realizado para posicionar a esta especie en Chile. De hecho, los programas de diversificación de las plantaciones forestales impulsadas en el país la señalan como una de las especies interesantes a considerar en las actuales forestaciones y, junto a otras especies, ha sido sujeto de bonificación especial por parte del Estado de Chile para fomentar su uso (CONAF 2006). En la actua- 
lidad, la superficie plantada con esta especie en Chile es cercana a 16.500 ha (INFOR-CONAF 1998), abarcando desde Maule hasta Magallanes (INFOR 2006).

Hasta la fecha, en las plantaciones de pino oregón del país no se habían detectado daños sanitarios de importancia. No obstante, a fines de octubre de 2006 se reportó al Laboratorio de Patología Forestal de la Universidad Austral de Chile una situación de anormalidad observada por primera vez y caracterizada por un amarillamiento y defoliación de árboles en plantaciones juveniles de esta especie. Estas plantaciones estaban localizadas en las cercanías de la ciudad de Valdivia, X Región, Chile. A partir de este hecho, el presente trabajo tuvo como objetivo determinar el agente causal de esta reciente situación de anormalidad sanitaria en pino oregón.

\section{MÉTODOS}

En un predio de uso agrícola, ganadero y forestal de 870 ha, ubicado a $35 \mathrm{~km}$ al noreste de la ciudad de Valdivia y localizado en el Área de Crecimiento 1-0-7 descrita por Schlatter et al. (1995), en octubre de 2006 se realizó una prospección y colecta de muestras en plantaciones jóvenes de pino oregón en las que estaban representadas edades de aproximadamente 2 a 12 años. En total se obtuvieron 42 muestras consistentes en ramillas extraídas tanto de la medianía como de la parte baja de la copa de los árboles examinados, abarcando todo el gradiente de sintomatología observado. Estas plantaciones totalizaban 30 ha distribuidas en aproximadamente veinte rodales distantes unos de otros por hasta $3 \mathrm{~km}$. Las muestras fueron procesadas en el Laboratorio de Patología Forestal de la Universidad Austral de Chile.

Para la determinación del agente causal del daño se efectuó la caracterización y medición de las diferentes estructuras fungosas encontradas, las cuales fueron montadas en agua destilada para su análisis microscópico. Para la identificación de éste, se contó con claves específicas y bibliografía ad hoc. En forma complementaria, una segunda prospección, a principios de noviembre de 2006, contempló la colecta de muestras de ramillas tomadas tanto de plantas $2 / 0$ de vivero (36 muestras) como de árboles de plantaciones de hasta 34 años de edad (56 muestras). Esta nueva prospección se realizó con el fin de constatar si la situación sanitaria se estaba produciendo en forma exclusiva en el primer predio reportado originalmente o era más extensa. La superficie total abarcada en esta última prospección estuvo contenida en un radio aproximado de $10 \mathrm{~km}$ a partir del margen noreste de la ciudad de Valdivia, y localizada en el Área de Crecimiento 1-0-9 descrita por Schlatter et al. (1995). La zona completa de prospección estuvo acotada, aproximadamente, por los paralelos $39^{\circ} 32^{\prime}-39^{\circ} 56^{\prime} \mathrm{S}$ y los meridianos $72^{\circ} 56^{\prime}-73^{\circ} 10^{\prime} \mathrm{O}$.

\section{RESULTADOS}

La prospección en el predio en el cual se estaba produciendo la situación de anormalidad reportada por primera vez permitió detectar que ésta se presentaba en árboles de pino oregón de todas las edades allí representadas (2 a 12 años). La sintomatología más destacable de esta anormalidad era la pérdida del color verde normal del follaje, el que en algunos casos presentaba un tono amarillento. En los casos más acentuados, las copas se mostraban de un tono amarillo-pardo, llegando en situaciones extremas a la defoliación prematura de los brotes laterales y apical. Cabe señalar que este cuadro sintomatológico era bastante variable de árbol en árbol (figura $1 \mathrm{~A}$, B). En la plantación más joven (2 años) la sintomatología más marcada se caracterizaba por la abundante presencia de manchas amarillo a amarillo-pardo, lo que le otorgaba un aspecto jaspeado a las acículas (figura $1 \mathrm{C}$ ).

A simple vista era muy difícil observar signos de la presencia de algún agente asociado a esta sintomatología. En los casos más acentuados, mediante una observación muy cuidadosa a ojo desnudo, se podían distinguir diminutas líneas de color negro, con aspecto de tizne u hollín, sólo en el envés de las acículas (figura $1 \mathrm{D}$ ). Mediante una lupa (x10) se pudo constatar que tales líneas correspondían a numerosas y pequeñísimas estructuras globosas de color negro distribuidas a lo largo de las dos bandas estomáticas. En laboratorio se pudo reconocer que estas estructuras se localizaban ordenadamente a lo largo de las líneas estomáticas, desarrollándose cada una de ellas exactamente sobre el estoma mismo (figura $1 \mathrm{E}, \mathrm{F})$.

Estas estructuras globosas correspondían a ascocarpos, de un tamaño de 55 a $80 \mu \mathrm{m}$ de diámetro, y en su interior contenían numerosos ascos ovoides a piriformes, de pared gruesa y consistente engrosada en la porción apical, ordenados en ramilletes, y medían aproximadamente 25 a $30 \mu \mathrm{m}$ de largo por 12 a $16 \mu \mathrm{m}$ de ancho (figura $1 \mathrm{G}, \mathrm{H}$ ). Estos ascos contenían en su interior ocho ascosporas bicelulares, hialinas, algo fusiformes, levemente acinturadas en la porción central donde se ubica el tabique que separa sus dos células, y de 12 a $16 \mu \mathrm{m}$ de largo por 5 a $6 \mu \mathrm{m}$ de ancho (figura $1 \mathrm{I}$ ).

Los ascocarpos fueron observados en todas las muestras colectadas, tanto en acículas que presentaban los síntomas ya descritos como en acículas completamente verdes (de color normal). En todas las ramillas analizadas los ascocarpos se encontraban presentes en acículas de todas las edades, incluidas las del último período vegetativo, con excepción de aquellas que estaban en el inicio de su formación en el período de muestreo. El número de ascocarpos por superficie foliar, estimado visualmente, era mayor en las acículas más antiguas.

El material vegetal colectado en las plantaciones de la prospección complementaria arrojó los mismos resultados ya descritos. Sin embargo, el hongo no estuvo pre- 


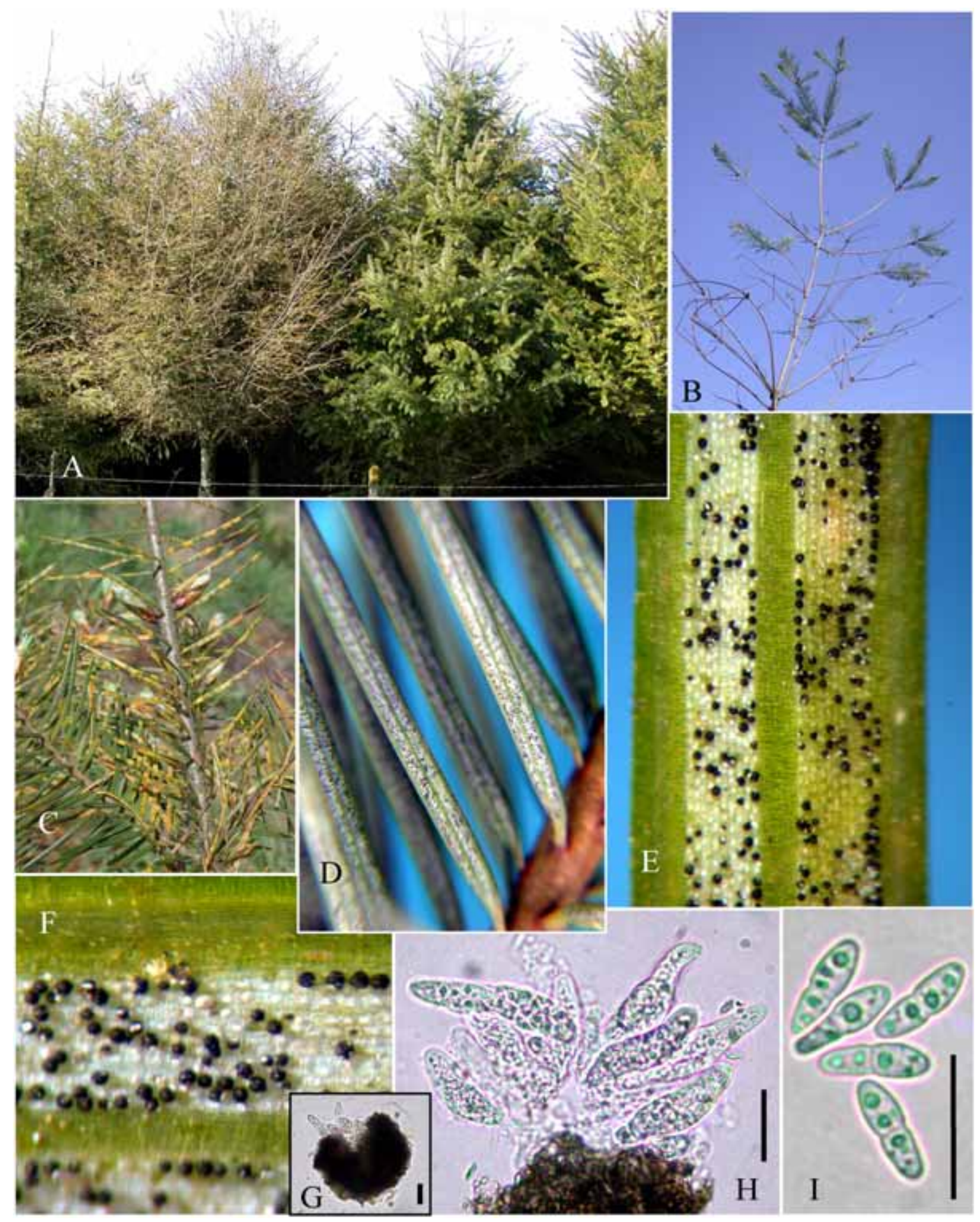

Figura 1. Síntomas y signos de la presencia de Phaeocryptopus gaeumannii en pino oregón. A) Árboles cuyas copas manifiestan diferentes grados de ataque foliar. B) Fuerte defoliación en la que permanecen sólo acículas del último período vegetativo. C) Jaspeado amarillo-pardo en acículas de plantas de dos años. D-E-F) Cara inferior de las acículas en las que se aprecian los diminutos ascocarpos a lo largo de ambas bandas estomáticas. G-H-I) Detalle de los ascocarpos, ascos y ascosporas típicas de P. gaeumannii. (La barra representa aproximadamente $20 \mu \mathrm{m}$ ).

Symptoms and signs of the presence of $P$. gaeumannii in Dougls-fir. A) Trees whose crowns show different degrees of attack to foliage. B) Hard defoliation in which only needles of the last vegetative period remain. C) Yellow-brown mottled in needles of plants of two years. D-E-F) Underside of infected needles in which the tiny ascocarps throughout both stomatics bands are appraised. G-H-I) Details of the ascocarps, ascus and ascospores of P. gaeumannii. (The bar represents approximately $20 \mu \mathrm{m}$ ). 
sente en todas las muestras del vivero, donde el número de ascocarpos por superficie foliar, estimado visualmente, fue algo menor que en las muestras de la primera prospección.

\section{DISCUSIÓN}

Tanto la sintomatología observada en el follaje de los árboles de pino oregón de las plantaciones con la situación de anormalidad sanitaria reportada (figura $1 \mathrm{~A}, \mathrm{~B}$, C), como las características microscópicas del hongo allí presente (figura $1 \mathrm{D}, \mathrm{E}, \mathrm{F}, \mathrm{G}, \mathrm{H}, \mathrm{I})$ y la asociación parásito-planta, permiten determinar que corresponde a un cuadro de enfermedad foliar causada por el hongo Phaeocryptopus gaeumannii (Rhode) Petrak. La sintomatología del daño encontrado y las características morfológicas del agente involucrado son, de una u otra forma, coincidentes con lo señalado por diversos autores respecto de una enfermedad en pino oregón, con similares síntomas y signos (Boyce 1948, 1961, Peace 1962, Hepting 1971, Butin y Zycha 1973, Dennis 1981, Butin 1983, 1989, Capretti et al. 1984, Funk 1985, Sinclair et al. 1987, Scharpf 1993).

Esta enfermedad fue reportada por primera vez en Suiza, en el año 1925, afectando a plantaciones de pino oregón. Luego, en 1928, fue observada en Inglaterra y en 1931 en Alemania (Peace 1962, Butin y Zycha 1973). Con leve diferencia cronológica respecto de los autores antes mencionados, Gäumann (1951) señala que, ya conocida esta enfermedad en Irlanda, Inglaterra y Suiza, alrededor de 1927 se expande como "pandemia" a toda Europa, lo que hizo poner en duda el cultivo del pino oregón en esos territorios. Alertados por la situación producida en Europa, se realizaron prospecciones en plantaciones localizadas en la región costera del Estado de Oregón (Estados Unidos), lugar originario del pino oregón, reportando en 1938 la presencia de esta enfermedad en dicho territorio (Peace 1962, Hansen et al. 2000). A la fecha, son numerosos los trabajos que reportan esta enfermedad en todo el rango natural del pino oregón y en aquellos lugares donde ha sido plantado como especie exótica, entre estos, países de Europa Occidental (incluido países escandinavos), Japón, Nueva Zelanda y Australia. Recientemente fue reportado en Turquía, afectando a plantaciones de pino oregón de 15 años (Temel et al. 2003).

Originalmente, esta enfermedad fue conocida en el idioma alemán como "Schweiser Douglasienschütte" ("defoliación suiza del pino oregón"), por ser justamente en ese país donde fue reportada por primera vez (Arx 1962) y de allí derivó su actual nombre en inglés "Swiss Needle Cast", por el cual se le conoce internacionalmente, y algunas veces nominado también por la sigla SNC. Esta enfermedad ha sido conocida en alemán también como "Russige Douglasienschütte" ("defoliación carbonosa del pino oregón") debido al aspecto negruzco o de hollín que se observa en el envés de las acículas afectadas por ella (Butin y Zycha 1973). En francés, esta enfermedad ha sido nominada como "La Rouille Souisse du Douglas" (Centre Technique du Genie Rural des Eaux et Forêst 1975, Lanier 1976, 1978). Este apelativo, "la roya suiza del pino oregón" no fue correctamente asignado, dado que el hongo que causa esta enfermedad, taxonómicamente, pertenece a la clase Ascomycetes (Orden Dothiorales) y no a la clase Basidiomycetes (Orden Uredinales), propia de las royas.

Como se pudo comprobar en las dos prospecciones realizadas, el efecto del hongo en diferentes rodales, como también en árboles individuales contiguos de un mismo rodal, es muy variable. Se encontraron árboles con follaje completamente verde y sano compartiendo espacio tanto con individuos que presentaban follaje levemente amarillento, como también con árboles que presentaban clorosis marcada, e incluso algunos que ya presentaban severa defoliación (figura $1 \mathrm{~A}$ ). Al parecer este comportamiento variable del pino oregón frente al ataque por $P$. gaeumannii podría tener relación con una aparente susceptibilidad individual de los árboles frente a la acción de este patógeno y es coincidente con lo observado en otras situaciones reportadas donde la apreciación de la severidad del daño ha resultado ser contradictoria (Peace 1962, Sinclair et al. 1987, Hood 1999).

En Norteamérica esta enfermedad ha llegado a constituirse en un problema tanto en plantaciones jóvenes de pino oregón como en el cultivo de esta especie para árboles de Navidad (Edmonds et al. 2000). En la costa del Estado de Oregón las plantaciones de 10 a 30 años de edad han resultado ser las más visiblemente afectadas por esta enfermedad (Hansen et al. 2000), pero existen reportes de ataques en árboles de hasta 55 años (Boyce 1948). En las prospecciones aquí realizadas fue posible detectar la presencia del hongo en plantaciones de todo el rango de edad disponible (plantas de vivero $2 / 0$ y plantaciones de 2 hasta 34 años), lo que sería coincidente con lo hasta ahora reportado en otras latitudes. También pareciera ser que el número de ascocarpos por superficie foliar es mayor en plantaciones jóvenes que en árboles más adultos y también que en plantas de vivero. Probablemente las aplicaciones más intensivas de biocidas y fertilizantes que se ejecutan en viveros puedan estar ejerciendo un cierto tipo de control frente al ataque de este hongo. Estos son temas que habrá que abordar convenientemente en futuros estudios.

En Europa, actualmente, esta enfermedad no representa un problema serio (Hood 1999). Sin embargo, en la costa norte del Estado de Oregón, corrientemente causa defoliaciones extremas, pérdida de crecimiento y mortalidad en plantaciones jóvenes, situación que se ha acentuado en los últimos años (Hansen et al. 2000).

La infección ocurre en acículas recién formadas y, en las prospecciones realizadas en plantaciones de los alre- 
dedores de Valdivia, se pudo comprobar que los síntomas de la enfermedad ya eran visibles en acículas formadas en el último período vegetativo (figura $1 \mathrm{~A}, \mathrm{C}$ ), lo que es coincidente con lo señalado por Sinclair et al. (1987). En general, los cuerpos fructíferos de $P$. gaeumannii (figura $1 \mathrm{D}, \mathrm{E}, \mathrm{F}, \mathrm{G}$ ), que corresponden a ascocarpos, denominados indistintamente como peritecios o pseudotecios por diferentes autores, son visibles en acículas de uno o más años (Butin y Zycha 1973, Funk 1985), pero algunas veces, como ocurrió en el material colectado en las presentes prospecciones, estos signos fueron también visibles en acículas formadas en el último período vegetativo; esto último es coincidente con lo señalado por Sinclair et al. (1987).

Peace (1962) señala que se han encontrado ascocarpos de este patógeno en pino oregón, en acículas de hasta siete años de edad. Pero la sola presencia de ascocarpos no implicaría necesariamente la caída de ellas y sí dependería de la abundancia en que los ascocarpos se presenten, ya que, según Hansen et al. (2000), la defoliación es proporcional al número de estomas ocluidos por los pseudotecios del hongo.

En casos individuales, donde el ataque fue más severo, los árboles retenían sólo las acículas del último período vegetativo (figura $1 \mathrm{~B}$ ), lo cual ya ha sido reportado para situaciones observadas en otras latitudes (Hansen et al. 2000). Respecto de esto último, cabe señalar que, según Boyce (1948, 1961), en defoliaciones producidas en años consecutivos, en las que sólo quedan las acículas de la temporada en curso, los árboles severamente afectados se tornan moribundos y finalmente mueren.

En cuanto a las condiciones que hacen propicio el que $P$. gaeumannii pueda cumplir su rol patogénico sobre pino oregón existe gran coincidencia entre lo señalado por diferentes autores. Todo indica que primaveras frías y húmedas favorecen el desarrollo de la enfermedad (Edmonds et al. 2000). Entre otras, probablemente hayan sido condiciones climáticas como éstas, registradas en los períodos primaverales de los últimos años, las que propiciaron el aparecimiento de dicha enfermedad en las plantaciones de pino oregón de esta región sur de Chile. Recientemente, Stone (2006) ha señalado que los incrementos de las temperaturas invernales y de las precipitaciones primaverales, que se registran desde la década de los 70 en el Pacífico Norte de los Estados Unidos, han influido en la distribución y severidad de esta enfermedad, y sugiere que estos dos últimos parámetros epidemiológicos tendrán un aumento como resultado del cambio climático, con consecuencias significativas para los bosques de esa región.

En Nueva Zelanda, desde que en sus plantaciones se reportara el aparecimiento de esta enfermedad en el año 1959, investigadores del New Zealand Forest Research Institute han realizado numerosos estudios para, entre otros, monitorear el ataque del hongo, conocer su biología, analizar aspectos epidemiológicos y de patogenici- dad, medir el impacto en el crecimiento de los árboles, probar métodos de control silviculturales y químicos, y establecer ensayos de resistencia y de tolerancia genética (Hood 2004). Del mismo modo, con el fin de comprender mejor la epidemiología de esta enfermedad y diseñar tratamientos silviculturales para maximizar la productividad del pino oregón y aminorar los problemas acarreados por esta epifitia en los bosques costeros de los estados de Oregón y Washington, en 1997 se constituyó la "Swiss Needle Cast Cooperative" en el Departamento de Ciencias Forestales de la Oregon State University (Mainwaring y Shaw 2005).

Para Chile el presente trabajo corresponde al primer reporte de la presencia de la enfermedad causada por $P$. gaeumannii en plantaciones de pino oregón del país. Al respecto, cabe destacar que la presencia consistente de este hongo en árboles de un amplio rango de edad (vivero hasta 34 años) y en acículas de un año y más, detectada en las prospecciones realizadas en rodales distantes unos de otros y en dos diferentes áreas de crecimiento que en conjunto abarcaron un radio aproximado de $15 \mathrm{~km}$, estaría señalando que este agente se habría establecido en el país desde hace ya algunos años. Sin lugar a dudas, frente a esta situación ya declarada, no cabe sino comenzar a trabajar interdisciplinariamente con el fin de realizar investigaciones básicas y aplicadas que permitan manejar adecuadamente la productividad de las plantaciones de pino oregón, conscientes de que en el futuro se deberá convivir con esta enfermedad.

\section{AGRADECIMIENTOS}

Al Director del Instituto de Silvicultura de la Facultad de Ciencias Forestales (Universidad Austral de Chile) Dr. Juan Schlatter V., por su apoyo financiero para que las imágenes de esta publicación pudiesen ser editadas a color.

\section{REFERENCIAS}

Arx v. JA. 1962. Beiträge zur Kryptogamenflora der Scheiz: Die Gattungen der didymosporen Pyrenomyceten. Warer, Berna, Suiza. 922 p. (Band 11, Heft 2).

Boyce JS. 1948. Forest Pathology. $2^{\text {a }}$ Ed., McGraw-Hill, New York. 550 p.

Boyce JS. 1961. Forest Pathology. $3^{\text {a }}$ Ed., McGraw-Hill, New York. 572 p.

Butin H. 1983. Krakheiten der Wald- und Parkbäume: Leitfaden zum Bestimmen von Baumkrankheiten. Georg Thime, Stuttgart. 172 p.

Butin H. 1989. Krakheiten der Wald- und Parkbäume: Diagnose-Biologie- Bekämfung. $2^{\mathrm{a}}$ Ed. Georg Thieme, Stuttgart. $216 \mathrm{p}$.

Butin H y H Zycha. 1973. Forstpathologie. Georg Thime, Stuttgart. $177 \mathrm{p}$. 
Capretti P, F Moriondo, A Ragazzi. 1984. La situazione fitosanitaria dei boschi alpini di Conifere. In Atti del $1^{\circ}$ Convengo Nazionale: Problemi Fitopatologici Delle Piante Forestali. Venecia-Mestre, Italia, 14 de diciembre 1984. p. 30-52.

Centre Technique du Genie Rural des Eaux et des Forêts. 1975. La Rouille Suisse du Douglas. Phaeocryptopus gaeumannii (Rhode) Petrak Dardelet, Grenoble, Francia. 3 p. (Information Technique Pour la Surveillance et la Proteccion Phytosanitaire de la Forêt, Fascicule II, Fichas ilustradas).

CONAF (Corporación Nacional Forestal, CL). 2006. Diversificación Forestal. Consultado el 01 de noviembre 2006. Disponible en http://www.conaf/?page=home/contents\& seccion_id=f14e75f4f8e2ba9ebd24f99ef18d3.

Davel M. 1998. Identificación y caracterización de zonas de crecimiento para pino oregón en la zona andina patagónica argentina. Tesis de Magíster en Ciencias. Valdivia, Chile. Facultad de Ciencias Forestales, Universidad Austral de Chile. 123 p.

Dennis RWG. 1981. British Ascomycetes. J Cramer, Vaduz, Alemania. 585 p.

Edmonds RL, JK Agee, RI Gara. 2000. Forest Health and Protection. McGraw-Hill, Boston. 60 p.

Funk A. 1985. Foliar fungi of western trees. Canadian Forestry Service, Pacific Forest Research Centre. 159 p. (BC-X-265).

Gäumann E. 1951. Pflanzliche Infektionslehre: Lehrbuch der allgemeinen Pflanzpathologie für Biologen, Landwirte, Förste und Pflanzenzüchter. Birhäusr, Basel, Suiza. 681 p.

Hansen EM, JK Stone, BR Capitano, P Rosso, W Sutton, L Winton, A Kanaskie, MG McWilliams. 2000. Incidence and Impact of Swiss Needle Cast in Forest Plantations of Douglas-fir in Coastal Oregon. Plant Dis. 84: 773-778.

Hood IA. 1999. Swiss Needle Cast Disease of Douglas-Fir: A review from New Zealand Perspective. 2 p. (Report ref 1999-01.). Consultado 08 noviembre 2006. Disponible en http://www.fhrc.org.nz/reports/report_details.asp? Report_ID=35.

Hood IA. 2004. Swiss Needle Cast Disease. The New Zealand Experience. Presentation. Conference: Growing DouglasFir in the Swiss Needle Cast Zone, Oregon State University. Oregon. 16 de noviembre, 2004. Consultado 01 noviembre 2006. Disponible en http://outreach.forestry. oregonstate.edu/snc/agenda.htm.
INFOR (Instituto Forestal, CL)-CONAF (Corporación Nacional Forestal, CL). 1998. Monografía de pino oregón, Pseudotsuga menziesii. Neuenschwander y Cruz, Santiago, Chile. 143 p.

INFOR (Instituto Forestal, CL). 2006. Estadísticas 20042005: Bosques plantados por especies según región. Consultado el 27 de noviembre 2006. Disponible en http://wwww.infor.cl/webinfor/estadisticas_Forestales/ 2004_2005/Recursos_Forestales/bso.

Lanier L, P Joly, P Bondoux, A Bellemère. 1976. Mycologie et Pathologie Forestières. Tomo II. Pathlogie Forestière. Masson, Paris. 478 p.

Lanier L, P Joly, P Bondoux, A Bellemère. 1978. Mycologie et Pathologie Forestières. Tomo I. Mycologie Forestière. Masson, Paris. 487 p.

Mainwaring D y D Shaw. (eds.) 2005. Swiss Needle Cast Cooperative. 2005 Annual Report. Department of Forest Science, Oregon State University. 60 p.

Peace TR. 1962. Pathology of trees and shrubs. With special reference to Britain. Clarendon, Oxford. 75 p.

Quiroz I, Y Rojas. 2003. Pino ponderosa y Pino oregón: Coníferas para el sur de Chile. Instituto Forestal, Sede Los Lagos. 316 p.

Scharpf RF. 1993. Diseases of Pacific Coast Conifers. U.S. Department of Agriculture, Forest Service, Washington. 199 p. (Agriculture Handbook No 521).

Schlatter JE, V Gerding, H Huber. 1995. Sistema de ordenamiento de la tierra: herramienta para la planificación forestal en la X Región. Universidad Austral de Chile, Facultad de Ingeniería Forestal, Valdivia, Chile. 93 p. (Serie Técnica).

Schütt P, H Weisgerber, HJ Schuck, UM Lang, B Stimm, A Roloff (eds.). 2004. Lexikon der Nadelbäume: Verbreitung-Beschreibung-Ökologie-Nutzung. Nikol, Hamburgo. $639 \mathrm{p}$.

Sinclair WA, HH Lyon, WT Johson. 1987. Diseases of trees and shrubs. Comstock, London. 574 p.

Stone JK. 2006. Predicting effects of climate change on swiss needle cast disease in the Pacific Northwest. 2006 Joint Annual Meeting, 29 julio-02 agosto, Québec, Canadá. CPS Abstracts of Presentation. Abstracts Book, 2006. p. 185.

Temel F, JK Stone, GR Jonson. 2003. First report of swiss needle cast caused by Phaeocryptopus gaeumannii on Douglas-Fir in Turkey. Plant Dis. 87: 1536. 